\title{
Radio Constraints of Dark Matter: A Review and Some Future Perspectives
}

\author{
Man Ho Chan
}

Citation: Chan, M.H. Radio Constraints of Dark Matter: A Review and Some Future Perspectives. Galaxies 2021, 9, 11. https://doi.org/ 10.3390/galaxies 9010011

Received: 9 December 2020 Accepted: 24 January 2021 Published: 28 January 2021

Publisher's Note: MDPI stays neutral with regard to jurisdictional clai$\mathrm{ms}$ in published maps and institutional affiliations.

Copyright: () 2021 by the author. Licensee MDPI, Basel, Switzerland. This article is an open access article distributed under the terms and conditions of the Creative Commons Attribution (CC BY) license (https:// creativecommons.org/licenses/by/ $4.0 /)$.
Department of Science and Environmental Studies, The Education University of Hong Kong, Tai Po, New Territories, Hong Kong, China; chanmh@eduhk.hk

\begin{abstract}
In the past few decades, many studies have analyzed the data of gamma-rays, X-rays, radio waves, electrons, positrons, anti-protons, and neutrinos to search for the signal of dark matter annihilation. In particular, analyzing radio data has been one of the most important and effective ways to constrain dark matter. In this article, we review the physics and the theoretical framework of using radio data to constrain annihilating dark matter. We also review some important radio constraints of annihilating dark matter and discuss the future perspectives of using radio detection to reveal the nature of dark matter.
\end{abstract}

Keywords: dark matter; radio observations

\section{Introduction}

Many theoretical models predict that dark matter (e.g., weakly interacting massive particles (WIMPs)) would self-annihilate to emit high-energy particles, such as gamma-rays, electron-positron pairs, and neutrinos [1]. In the past decade, many detectors started to collect observational data to constrain annihilating dark matter. For example, the gammaray data collected by the Fermi-LAThave been used to investigate the possible signal of dark matter [2-7]. Besides, the AMSand DAMPEdetectors were launched to detect any cosmic-ray excess in our galaxy [8-11]. Some recent studies have claimed some possible excess of gamma-rays or cosmic-rays originated from dark matter annihilation $[2,4,9,10]$. However, the claims are still controversial because the systematic uncertainties involved are not negligible [12,13].

Apart from gamma-ray and cosmic-ray detections, radio detection is also a robust way to examine the alleged signal of dark matter annihilation. The high-energy electrons and positrons produced from dark matter annihilation would emit synchrotron radiation in the radio bands when there is a strong magnetic field. Since many radio telescopes and interferometers have very good sensitivity and resolution, any synchrotron signals due to dark matter annihilation from extragalactic origins could still be detected. For example, earlier studies have examined the radio signals of our galaxy and its satellite galaxies to constrain dark matter [14-26]. Later, radio signals from other nearby galaxies such as the M31 [27-29], M33 [30,31], NGC 1569 [32], and NGC 2976 [33] galaxies were also investigated in constraining dark matter. Besides galactic radio signals, radio signals from galaxy clusters have been investigated [34-39]. Some other related studies such as the radio excess of extragalactic sources (e.g., ARCADEexcess) [40] have also been discussed in constraining dark matter. The constraints obtained by these studies are complementary to the gamma-ray, cosmic-ray, and neutrino constraints.

In this article, we first review the basic physics and the theoretical framework of the dark matter annihilation model. Then, we review some important constraints of dark matter annihilation based on radio detections and discuss their limitations. We also discuss some intriguing results obtained recently, which suggest some possible signals of dark matter. Lastly, we discuss some future perspectives in using radio telescopes to solve the dark matter mystery. 


\section{Radio Emissions Due to Dark Matter Annihilation}

If dark matter can self-annihilate, it can produce a large number of high-energy photons, electrons, positrons, and neutrinos via different possible annihilation channels. The self-annihilation rate depends on the average annihilation cross-section $\langle\sigma v\rangle$ and the dark matter mass $m_{D M}$. The energy spectrum $d N / d E$ for each kind of high-energy particle and each annihilation channel can be calculated numerically (i.e., the injected energy spectrum) [41]. The electrons and positrons injected would cool down during diffusion, which can be described by the cooling-diffusion equation [34,42]:

$$
\frac{\partial}{\partial t} \frac{d n_{e}}{d E}=\nabla\left[D(E, r) \nabla \frac{d n_{e}}{d E}\right]+\frac{\partial}{\partial E}\left[b(E) \frac{d n_{e}}{d E}\right]+Q(E, r),
$$

where $d n_{e} / d E$ is the equilibrium electron/positron density spectrum, $D(E, r)$ is the spatial diffusion coefficient, $b(E)$ is the cooling rate, and $Q(E, r)=\left(\langle\sigma v\rangle \rho_{D M}^{2} / 2 m_{D M}^{2}\right)(d N / d E)$ is the dark matter annihilation source term. Some other terms like the convection term could also be involved in the above cooling-diffusion equation. However, the convection effect is important only if we consider the small region near the center of a galaxy. In the following, we focus on discussing a large region of interest (e.g., $R>1 \mathrm{kpc}$ ) so that Equation (1) would be sufficient to describe the diffusion and cooling processes. This equation can be generally solved by public numerical codes like GALPROP [43], Dragon [44], PICARD [45], and USINE [46] or the Green's function method [34,42,47]. The equilibrium electron/positron density spectrum can be written in terms of Green's function [47]:

$$
\frac{d n_{e}}{d E}=\frac{1}{b(E)} \int_{E}^{m_{D M}} G(r, \Delta u) Q\left(E^{\prime}, r\right) d E^{\prime}
$$

with:

$$
G(r, \Delta u)=\frac{1}{4 \pi \sqrt{\Delta u}} \sum_{i=-\infty}^{\infty}(-1)^{i} \int_{0}^{R} \frac{r^{\prime} d r^{\prime}}{r_{i}}\left[\frac{\rho_{D M}\left(r^{\prime}\right)}{\rho_{D M}(r)}\right]^{2}\left[e^{-\left(r^{\prime}-r_{i}\right)^{2} / 4 \Delta u}-e^{-\left(r^{\prime}+r_{i}\right)^{2} / 4 \Delta u}\right],
$$

where $r_{i}=(-1)^{i} r+2 i R$ and $\Delta u=\int_{E}^{E^{\prime}}[D(\tilde{E}) / b(\tilde{E})] d \tilde{E}$.

Nevertheless, if diffusion is not very significant (the diffusion timescale $\tau_{d}=R^{2} / D_{0}$ is much larger than the cooling timescale $\tau_{c}=E / b(E)$, and $D_{0}$ is the diffusion coefficient), Equation (1) can be analytically simplified to give an equilibrium energy spectrum [34,35]:

$$
\frac{d n_{e}}{d E}=\frac{\langle\sigma v\rangle \rho_{D M}^{2}}{2 m_{D M}^{2} b(E)} \int_{E}^{m_{D M}} d E^{\prime} \frac{d N_{e}}{d E^{\prime}}
$$

This would be a good assumption if the cooling rate is sufficiently high (e.g., average magnetic field strength $B \geq 5 \mu \mathrm{G}$ ) and the size of the structure is large (e.g., $R \geq 10 \mathrm{kpc}$ ) so that we have $\tau_{d} \gg \tau_{c}$. In general, this is a very good approximation for galaxy clusters. However, such an assumption might be critical to a normal galaxy (quite bad for dwarf galaxies). The results could significantly depend on the functional form of $D(E, r)$ and the value of the diffusion coefficient $D_{0}$, which are not easy to determine. Therefore, for simplifying the discussions and getting a more analytic picture, we omit the effect of diffusion in the following.

For the injected high-energy electrons and positrons $(E \sim 1 \mathrm{GeV})$, the major cooling mechanisms are synchrotron cooling, inverse Compton scattering (ICS), Bremsstrahlung cooling, and Coulomb loss [34]. Generally speaking, the cooling rate is dominated by synchrotron and ICS cooling in galaxies and galaxy clusters. The cooling rate (in $10^{-16} \mathrm{GeV} / \mathrm{s}$ ) can be explicitly given by [27]:

$$
b(E)=\left[0.025\left(\frac{B}{1 \mu \mathrm{G}}\right)^{2}+0.76\left(\frac{U_{p h}}{1 \mathrm{eV} / \mathrm{cm}^{3}}\right)\right]\left(\frac{E}{1 \mathrm{GeV}}\right)^{2},
$$


where $U_{p h}$ is the photon energy density. Note that, in general, the magnetic field strength $B$ and the energy density $U_{p h}$ are position-dependent so that the cooling function $b(E)$ should also be a function of $r$ as well. The typical magnetic field strength of a galaxy or a galaxy cluster is $B \sim 1-10 \mu \mathrm{G}$.

The radio flux density contributed by dark matter annihilation originates from the cooling of high-energy electrons and positrons due to synchrotron emission. The synchrotron power at radio frequency $v$ is given by $[48,49]$ :

$$
P_{D M}(E, v, \vec{r})=\frac{\sqrt{3} e^{3}}{m_{e} c^{2}} B(\vec{r}) F\left(v / v_{c}\right)
$$

where $v_{c}$ is the critical synchrotron frequency and $F(x)=x \int_{x}^{\infty} K_{5 / 3}(y) d y \approx 1.25 x^{1 / 3} e^{-x}$ $\left(648+x^{2}\right)^{1 / 12}$ is the synchrotron kernel function. Since the radio emissivity is mainly determined by the peak radio frequency (monochromatic approximation), the kernel function can be approximated by $F(x) \approx 8 \pi \delta(x-1 / 3) / 9 \sqrt{3}$, and the total radio flux density contributed by dark matter annihilation at frequency $v$ is given by $[48,49]$ :

$$
S_{D M} \approx \frac{1}{4 \pi v D_{L}^{2}}\left[\frac{9 \sqrt{3}\langle\sigma v\rangle}{2 m^{2}(1+C)} E(v) Y\left(v, m_{D M}\right) \int \rho_{D M}^{2} d V\right],
$$

where $C$ is the ICS correction factor (ratio of the ICS cooling rate to the synchrotron cooling rate), $D_{L}$ is the distance to the structure, $E(v)=14.6(v / \mathrm{GHz})^{1 / 2}(B / \mu \mathrm{G})^{-1 / 2} \mathrm{GeV}$, and $Y\left(v, m_{D M}\right)=\int_{E}^{m_{D M}}\left(d N / d E^{\prime}\right) d E^{\prime}$. Here, a point-source approximation has been adopted in the calculation of the total radio flux density (not in radio observations). This is true if the angular size of the structure is less than $1^{\circ}$ [50].

If dark matter particles were produced thermally in the early universe, the simplest model in standard cosmology predicts that the annihilation cross-section is $\langle\sigma v\rangle \approx 2.2 \times 10^{-26} \mathrm{~cm}^{3} / \mathrm{s}$ for $m_{D M} \geq 10 \mathrm{GeV}$ [51]. Therefore, testing this specific scenario is extremely important. However, some models suggest that the annihilation cross-section of thermal dark matter could depend on other factors like the velocity dispersion of dark matter particles $[52,53]$. Moreover, if dark matter particles are not thermal relic particles, some hypothetical models or scenarios have predicted other values of the non-thermal annihilation cross-section [54-56]. Therefore, in view of these models, the annihilation cross-section would become an extra parameter to constrain. Placing a constraint on the annihilation cross-section would be useful to test for these hypothetical models and scenarios. To model the dark matter density profile, the Navarro-Frenk-White (NFW) density profile is usually assumed [57]:

$$
\rho_{D M}=\frac{\rho_{s} r_{s}^{3}}{r\left(r+r_{s}\right)^{2}}
$$

where $\rho_{s}$ and $r_{s}$ are the scale density and scale radius, respectively. Besides the NFW profile, some other profiles may also be used for specific galaxies, such as the Burkert profile or the pseudo-isothermal profile [41]. For galaxy clusters, the hydrostatic density profile would be used as well $[37,58]$. The hydrostatic density profile depends on the thermal properties of the hot gas, which can be obtained easily from X-ray observations [58].

Furthermore, dark matter substructures in galaxies and galaxy clusters can significantly boost the annihilation signals because the annihilation rate is directly proportional to $\rho_{D M}^{2}$. Numerical simulations have provided some empirical relations for galaxies [59] and galaxy clusters [60] to quantify the effects of the annihilation boost. These relations can be applied directly to predict the enhanced dark matter annihilation signal. However, the empirical relations are somewhat model dependent, and they depend on the virial mass of a structure, which may have a large uncertainty in the value (e.g., virial mass of the M31 galaxy $\left.=(7-24) \times 10^{11} M_{\odot}[61]\right)$. For example, the empirical relation for galaxies in [59] assumed the NFW dark matter density profile, while the relation for galaxy clusters 
in [60] followed a parametrized form of the mass-concentration relation. Furthermore, both relations depend on the values of the slope of the halo mass function $[59,60]$. The typical values of the boost factors for galaxies and galaxy clusters are $\sim 1-10$ and $\sim 30-70$, respectively [59].

\section{Results}

The total radio flux density contributed by dark matter annihilation $S_{D M}$ depends on the annihilation cross-section $\langle\sigma v\rangle$, dark matter density profile $\rho_{D M}$, magnetic field strength $B$, and dark matter mass $m_{D M}$. The dark matter density profile and the magnetic field strength could be modeled independently by other observations. For example, the dark matter density profile can be probed from galactic rotation curves or hot gas distribution in galaxy clusters. For the magnetic field strength, it could be obtained by Faraday rotation measures (see [62]) or empirical relations based on numerical simulations [63,64]. Therefore, by setting $S_{D M}$ smaller than the observed total radio flux density of a structure, we can obtain some constraints of $\langle\sigma v\rangle$ and $m_{D M}$. In the following, we review some previous constraints obtained by using the radio data of some nearby galaxies and galaxy clusters.

\subsection{Galaxies}

Early studies have used the data of our Milky Way galaxy to constrain dark matter annihilation. For example, the studies in $[14,15]$ used the Milky Way radio data to constrain the annihilation of WIMPs or neutralinos with mass $\sim 100 \mathrm{GeV}$. Later, the radio data of the Milky Way satellite galaxies such as the Large Magellanic Cloud were also investigated $[16,17,19]$. These studies provided some good constraints for $m_{D M} \sim 50 \mathrm{GeV}[16,19]$. Generally speaking, the thermal dark matter scenario is allowed by the constraints for masses above $\sim 50-100 \mathrm{GeV}$. On the other hand, the measurements from the Wilkinson Microwave Anisotropy Probe (WMAP) and the Planck detector have revealed some excess of microwave emission from the region around the center of our galaxy (the WMAP-Planck haze), which may originate from dark matter annihilation $[65,66]$. The best-fit dark matter mass could be of the order $\sim 100 \mathrm{GeV}$ or larger [65]. However, using Milky Way radio data to constrain dark matter suffers from some difficulties. First of all, the diffusion is quite important outside the galactic center region. There are many sets of diffusion models and diffusion parameters suggested to model the diffusion process [41,67]. The uncertainties of the parameters can be a factor of 10 or larger. Some studies have discussed the impact of these uncertainties based on large-scale observations of the Milky Way [68]. If we focus on the galactic center region instead, the effect of advection would be important, and the uncertainty of the magnetic field profile would be very large [21]. Besides, a dark matter density spike might be formed due to the supermassive black hole, so that the dark matter annihilation rate would be significantly enhanced $[69,70]$. This uncertainty could be quite significant. Similar problems are also encountered for analyzing the WMAP-Planck haze [71]. On the other hand, using the radio data of Milky Way dwarf galaxies to constrain annihilating dark matter is also not very good. This is because these dwarf galaxies are too small, so the diffusion effect is very important, which would largely suppress the radio flux emitted. Although using radio data of some local dwarf galaxies may be able to give stringent constraints based on some average or canonical values of the parameters (e.g., see [22]), the uncertainties associated with the involved parameters are usually very large (more than a factor of 10 in uncertainties). Therefore, the resultant constraints obtained would be less certain. Generally speaking, the constraints obtained by using the gamma-ray data of Milky Way dwarf galaxies have less uncertainties and thus are more robust [3].

Besides the Milky Way galaxy and its satellite galaxies, many studies have focused on the nearby galaxies M31 and M33 to constrain dark matter annihilation. For the M31 galaxy, Egorov and Pierpaoli [27] performed a comprehensive analysis of the radio survey data of the central region. Including possible uncertainties, the allowed dark matter mass could range from $m_{D M} \sim 20 \mathrm{GeV}$ to $200 \mathrm{GeV}$ [27]. Later, the study in [28] considered the radio data of a larger region $(\approx 17 \mathrm{kpc})$ of the M31 galaxy obtained by the Westerbork 
Synthesis Radio Telescope (WSRT) in [72]. The upper limits of $\langle\sigma v\rangle$ for six annihilation channels were obtained for $m_{D M}=10-1000 \mathrm{GeV}$ (see Figure 1) [28]. Recently, by observing the data of the M31 galaxy using the Nanshan Radio Telescope (NSRT) at C-band frequency $(v=4.6-5.0 \mathrm{GHz})$, much more stringent constraints were obtained for $m_{D M} \geq 100 \mathrm{GeV}$ [29]. Similar analyses have been performed for the M33 galaxy as well $[30,31]$. Note that the constraints in $[28,29,31]$ were obtained without considering the effect of diffusion. The actual lower limits of $m_{D M}$ should be somewhat smaller and depend on the actual diffusion parameters for the M31 and M33 galaxies.
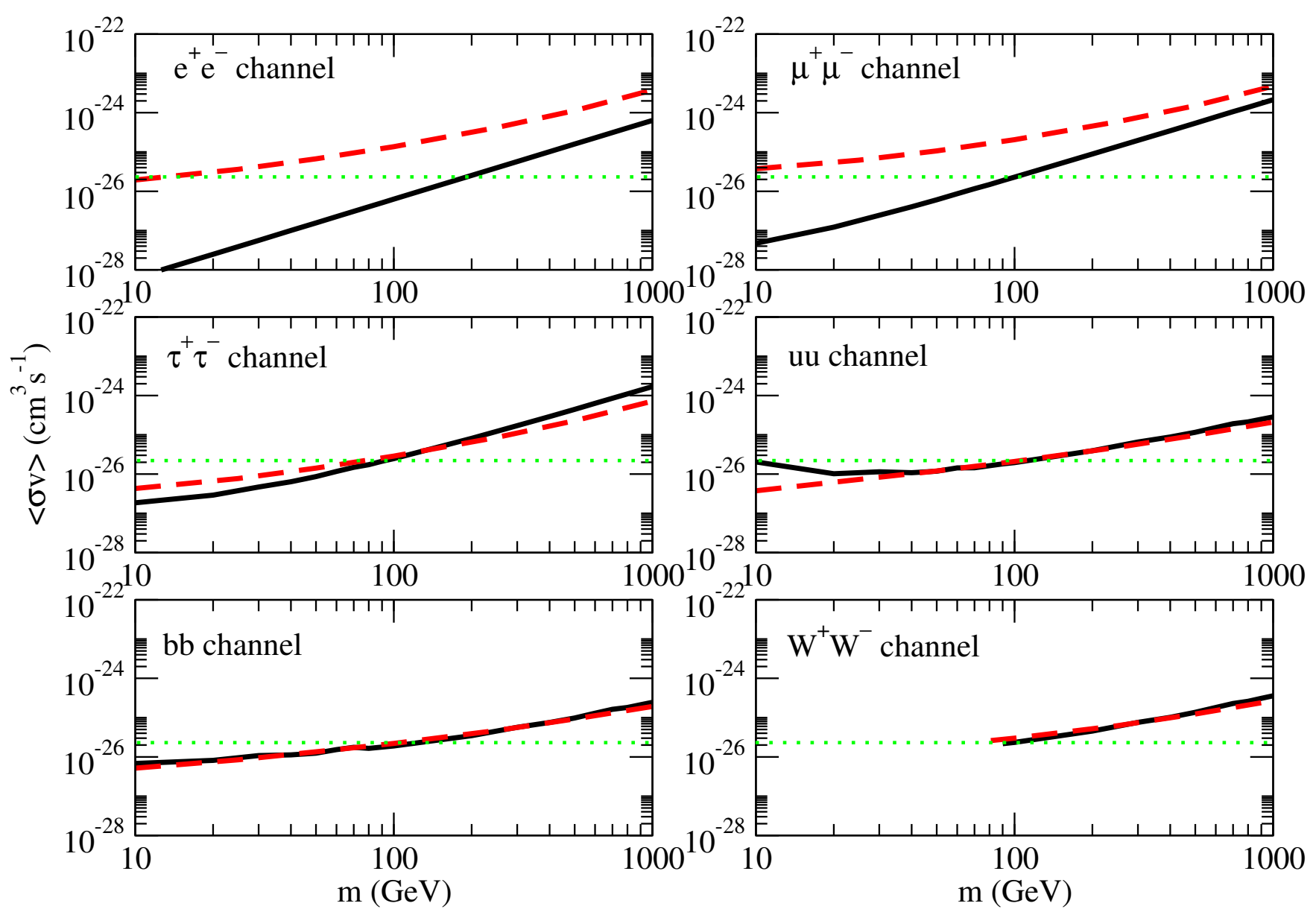

Figure 1. The black solid lines represent the upper limits of the annihilation cross-sections for six popular annihilation channels based on our previous radio analysis [28]. The red dashed lines are the corresponding upper limits based on the gamma-ray analysis of the Milky Way dwarf spheroidal galaxies [3]. The green dotted lines indicate the thermal relic annihilation cross-section $\langle\sigma v\rangle=2.2 \times 10^{-26} \mathrm{~cm}^{3} \mathrm{~s}^{-1}$.

There are some nearby galaxies that are also suitable for constraining annihilating dark matter. For example, the study in [33] used the radio data of the NGC 2976 galaxy at $v=1.43 \mathrm{GHz}, 4.85 \mathrm{GHz}$, and $8.35 \mathrm{GHz}$ to constrain dark matter. The constraints are generally tighter than the gamma-ray constraints for $m_{D M} \geq 100 \mathrm{GeV}$. Furthermore, a recent study using the radio data of the NGC 4214 galaxy provided stringent constraints for $m_{D M} \geq 200 \mathrm{GeV}$ [73]. Therefore, many extragalactic targets are suitable for performing radio analyses to constrain annihilating dark matter.

Besides focusing on individual extragalactic targets, some studies have analyzed the alleged extragalactic radio excess to constrain dark matter. For example, the study in [40] discussed the possibility of the dark matter interpretation of the radio excess detected by the ARCADE observations. However, such a possibility has been challenged by other recent proposals such as cluster mergers [74] and fast radio transients [75]. Furthermore, the pa- 
rameters obtained in [40] are in tension with the AMS data [76]. Therefore, fewer studies are now focusing on the effect of annihilating dark matter on extragalactic radio excess.

Note that using the galactic radio data may involve some systematic uncertainties. First of all, the magnetic field strength profile is one of the major systematic uncertainties. Some studies assume an exponential profile [27], while some studies assume a constant profile (average values) $[28,29,31]$. Furthermore, as mentioned above, the diffusion effect of the high-energy electrons and positrons is difficult to model precisely. Even if we fully follow the benchmark diffusion scenario by taking the diffusion coefficient $D_{0}$ and the diffusion index into account, the actual values of these parameters are not quite certain. It is also not very clear whether we can adopt a simple parametrized form of diffusion model to mimic the actual diffusion effect. Theoretical studies have proposed different possible versions of diffusion models (e.g., Kolmogorov model, Boehm model), which might be applicable to different regions [67]. Although it is true that we should consider the diffusion effect, the resultant constraints would significantly depend on the diffusion models considered and the model-dependent diffusion parameters. The diffusion parameters for any particular diffusion model are usually determined by cosmic-ray data. Furthermore, determining the size of the diffusive halo is also important. For example, recent studies in $[77,78]$ constrained the relevant diffusion parameters and their uncertainties by cosmic-ray data. However, for other galaxies, these propagation parameters are difficult to measure and determine.

Some studies assume that the diffusion is not important and apply Equation (7) to estimate the synchrotron fluxes for some galaxies with a large magnetic field strength (e.g., the results in Figure 1) $[28,29,31]$. This assumption is good if the magnetic field strength is sufficiently large so that the synchrotron cooling is efficient enough to suppress the diffusion effect. The final constraints would not depend on any unjustified diffusion scenarios or diffusion parameters. However, generally speaking, the lower limits obtained from these studies are overestimated as suggested in [79]. Based on the available galactic radio data, a large parameter space is still allowed for the specific thermal dark matter scenario, especially for the popular $b \bar{b}$ channel and $m_{D M}<100 \mathrm{GeV}$ [79]. Overall speaking, using the radio data of galaxies may suffer from large systematic uncertainties, which may give less robust constraints on dark matter mass and the annihilation cross-section.

\subsection{Galaxy Clusters}

Galaxy clusters are also very good targets for analyses. Most of them are dark matter rich, and the effect of substructures may greatly enhance the radio signals due to dark matter annihilation $[60,80]$. Furthermore, the diffusion coefficient in a typical galaxy cluster is $\sim 10^{30} \mathrm{~cm}^{2} / \mathrm{s}$ [67], which gives a very long diffusion timescale. In other words, the diffusion is insignificant in galaxy clusters so that the synchrotron radiation would not be suppressed and the results would not depend on the uncertain diffusion scenario. Besides, numerical simulations show that the magnetic field profile in a galaxy cluster traces the thermal electron density profile $\left(B(r) \propto[n(r)]^{\eta}\right.$, with $\left.\eta=0.5-1.0\right)[63,81]$, where the thermal electron density profile can be best modeled by the observed X-ray surface brightness profile (the $\beta$ model) [58]. The central magnetic field strength $B_{0} \sim 10 \mu \mathrm{G}$ can also be estimated by the thermal properties of the hot gas $[63,64]$. Therefore, fewer uncertainties would be involved compared with using the galactic radio data. Moreover, the radio contribution of the hot gas is insignificant (less than 1\%) [37]. Hence, the major radio emissions in a galaxy cluster originate from two components only: dark matter annihilation and the background cosmic-ray contribution.

Many earlier studies focused on large galaxy clusters such as the Coma cluster to constrain dark matter annihilation [34-36]. However, the resultant constraints of annihilating dark matter are not very stringent. It is because the distance to a nearby galaxy cluster is usually very large $\left(D_{L} \geq 10 \mathrm{Mpc}\right)$. This gives a very small total radio flux reaching radio telescopes. Such a problem can only be tackled if we have radio telescopes with very high sensitivity. 
Recently, some studies have started to model the background cosmic-ray contributions [37-39]. If we could eliminate the background cosmic-ray contributions from the total radio emission, we could get much tighter constraints for annihilating dark matter. The cosmic-ray models in galaxy clusters have been developed for a few decades, which include the primary emission model [82-84], secondary emission model [85,86], first-order Fermi acceleration model (Fermi-I) [87,88], second-order Fermi acceleration model (Fermi-II) [89-91], and adiabatic compression model [92,93]. These models can predict the functional forms of the possible frequency spectra of the background cosmic-ray emission in galaxy clusters. For example, the secondary emission model predicts a powerlaw frequency spectrum $\left(S(v) \propto v^{-\alpha}\right)$, while the Fermi-II model predicts a power-law with a high-frequency cutoff $\left(S(v) \propto v^{-\alpha} e^{-\beta v}\right)$ in the spectral shape [90]. In particular, previous studies have shown that the Fermi-II model and the adiabatic compression model can best describe the radio cosmic-ray frequency spectra of the Coma cluster [90] and the Abell 4038 cluster [94].

Based on our understanding of the cosmic-ray emission frequency spectrum, we can theoretically eliminate the cosmic-ray contribution from the total radio frequency spectrum. If there is some "radio excess" after subtracting the best-fit cosmic-ray contribution from the total radio frequency spectrum, then we can test for the dark matter annihilation model and get a best-fit dark matter contribution. In general, the spectrum of the cosmic-ray contribution would give an almost constant spectral index in the low frequency regime. The dark matter contribution would steepen the spectral index at low frequencies so that a smooth "spectral break" exists in the radio frequency spectrum. Moreover, at high frequencies, some cosmic-ray models (e.g., Fermi-II) would show an exponential decline in the spectrum. The dark matter contribution would suppress the decline in the highfrequency regime (an inflection point exists) and reveal a possible signature of dark matter annihilation signal.

This method can give more stringent constraints for dark matter mass and annihilation cross-section. More importantly, it can reveal some possible signals of dark matter annihilation if there exists a "radio excess" in the frequency spectrum. It can provide an indirect way to search for the signal of dark matter annihilation. Recent studies using the total radio flux density spectra of the Ophiuchus cluster (the central region only) [95], the Abell 697 cluster [96], and the Abell 4038 cluster [97] revealed some possible signals of dark matter annihilation (see Figures 2-4) [37-39]. Assuming the thermal annihilation cross-section, the best-fit dark matter mass is $m_{D M} \sim 30-150 \mathrm{GeV}$ for four representative annihilation channels $\left(e^{+} e^{-}, \mu^{+} \mu^{-}, \tau^{+} \tau^{-}\right.$, and $\left.b \bar{b}\right)$. This range is consistent with the results in many recent gamma-ray studies (e.g., $m_{D M} \approx 30-35 \mathrm{GeV}$ via $b \bar{b}[98] ; m_{D M} \approx 30-40 \mathrm{GeV}$ via $b \bar{b}[4] ; m_{D M} \sim 80 \mathrm{GeV}$ via $\mu^{+} \mu^{-}$[2]) and anti-proton studies $\left(m_{D M}=48-67 \mathrm{GeV}\right.$ via $b \bar{b}$ ) [99]. Note that for the Abell 4038 cluster, the cosmic-ray model can give a good fit for the radio relic (the major cosmic-ray source) only, while the two-component model (dark matter plus cosmic-ray contribution) can give a better fit for the total radio flux density spectrum (see the discussion in [39]).

Overall, in comparison, it seems that using the radio data of galaxy clusters is better than using the radio data of galaxies to constrain annihilating dark matter. This is because the systematic uncertainties of the diffusion effect would not be encountered in galaxy clusters. Nevertheless, the distances to galaxy clusters are usually larger so that the resultant radio density fluxes observed would be much smaller. Therefore, to get a more precise analysis using galaxy clusters, radio telescopes with very high sensitivity (e.g., radio interferometer) are required to perform the task for constraining annihilating dark matter. 


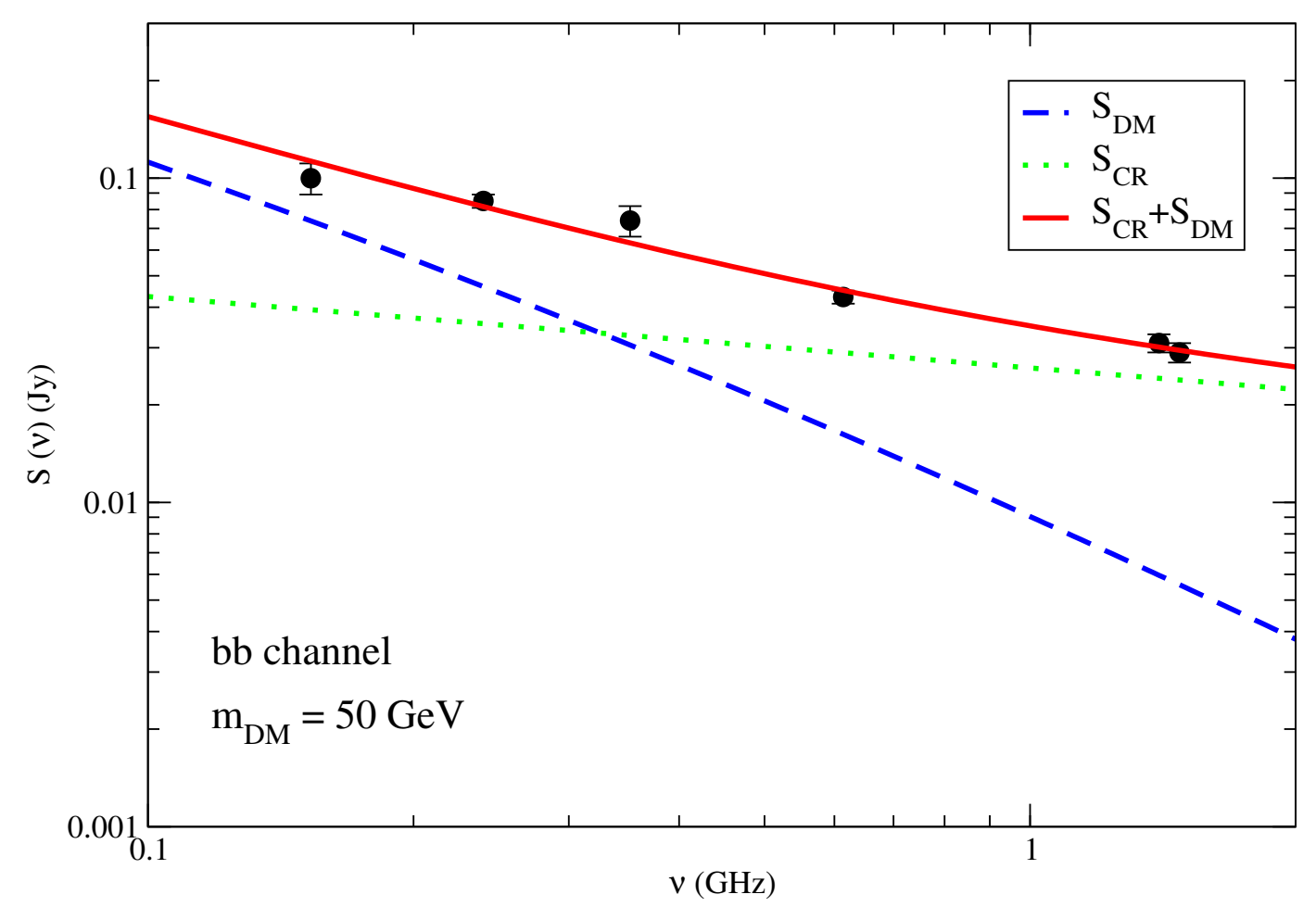

Figure 2. The best-fit dark matter scenario for the central Ophiuchus cluster radio data $\left(m_{D M}=50 \mathrm{GeV}\right.$ via the $b \bar{b}$ channel) [37]. The red solid line, green dotted line, and blue dashed line represent the total predicted radio flux $S(v)$, the cosmic-ray contribution (power-law form), and the dark matter contribution, respectively. The data with error bars were extracted from [95].

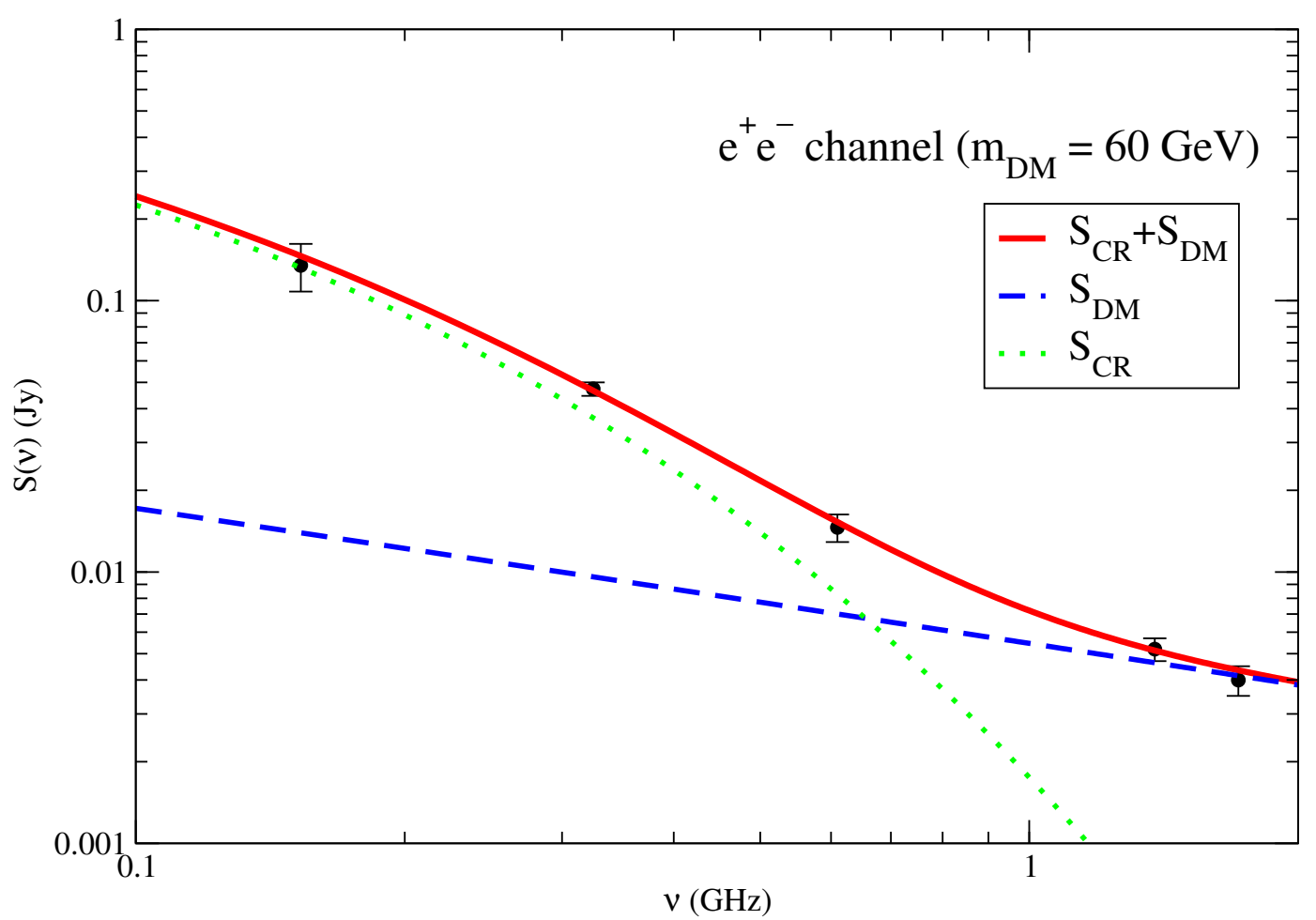

Figure 3. The best-fit dark matter scenario for the Abell 697 cluster radio data $\left(m_{D M}=60 \mathrm{GeV}\right.$ via the $e^{+} e^{-}$channel) [38]. The red solid line, green dotted line, and blue dashed line represent the total predicted radio flux $S(v)$, the cosmic-ray contribution (Fermi-II model), and the dark matter contribution, respectively. The data with error bars were extracted from [96]. 

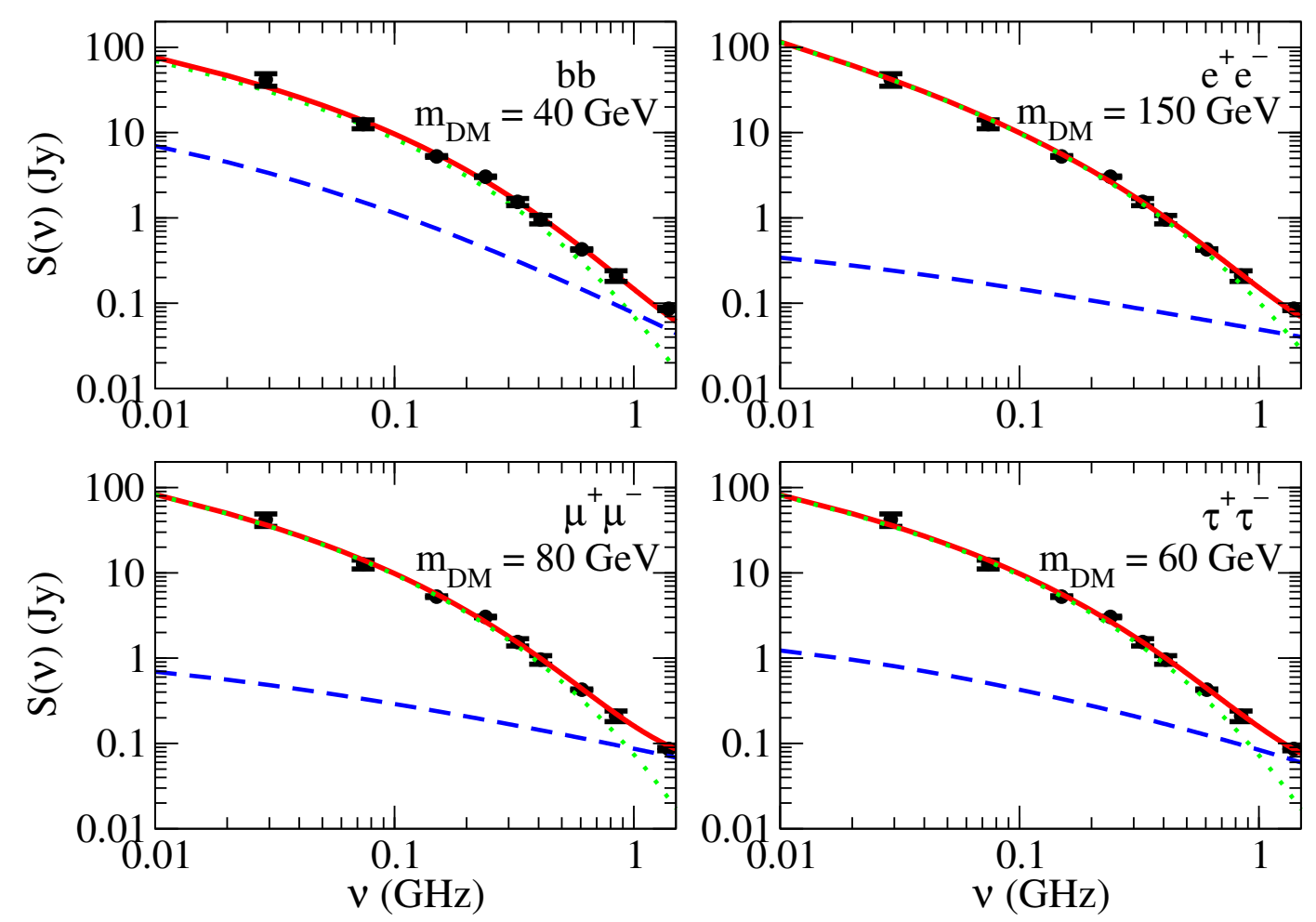

Figure 4. The best-fit dark matter scenario for the Abell 4038 cluster radio data [39]. The red solid line, green dotted line, and blue dashed line represent the total predicted radio flux $S(v)$, the cosmic-ray contribution (adiabatic compression model), and the dark matter contribution, respectively. The data with error bars were extracted from [97].

\section{Future Perspectives}

As mentioned above, recent studies have shown that using the frequency spectra of galaxy clusters would be good to constrain and search for the signal of dark matter annihilation. However, the archival data used in those studies mainly contain low-frequency data (e.g., 0.1-1.4 GHz) [37-39]. Generally speaking, a radio frequency spectrum with a wide range of frequencies (e.g., $0.1-10 \mathrm{GHz}$ ) is more likely to manifest a clearer signal of dark matter annihilation. Nevertheless, this requires new observations using radio telescopes with very high sensitivity and high observing frequencies (e.g., $v \geq 5 \mathrm{GHz}$ ). Some new and planned radio telescopes (e.g., FAST) or interferometers (e.g., SKA) may be able to fulfill the task $[47,100]$.

On the other hand, the radio emission in a galaxy or galaxy cluster depends on both frequency and position. Besides the frequency spectrum, the radial emission profile can also be used to constrain dark matter. Since the dark matter distribution is spherically symmetric, the dark matter radio emission profile would depend on the radial distance from the center only. If we can consider some specific regions (e.g., central bulge region of a galaxy) in which the background astrophysical radio emission (e.g., pulsar emission) is also close to spherically symmetric, then we can use the radial emission profile to constrain dark matter. By using the observed radio map of a central region, we can take the azimuthal averaging of the radio flux density in concentric bins for different angular radii from the center to get the radial emission profile. Then, by assuming the background astrophysical emission traces the stellar distribution (only has radial dependence), we can model the radial emission profile of the background emission. By subtracting the background emission from the total radial emission profile, the excess remaining would be the possible contribution by dark matter annihilation.

In fact, the radial emission profile of gamma-ray emission in our galaxy has also been considered previously [2,4]. However, it is now controversial to conclude whether the gamma-ray emission profile in our galaxy traces the dark matter density profile or 
not [101]. A recent study used the radio map of the central region of the M31 galaxy obtained in [62] to constrain dark matter [102]. Although the dark matter signal is not very significant, adding the dark matter contribution would give a better fit for the radio flux emission profile data (see Figure 5) [102]. The best-fit dark matter mass $m_{D M} \sim 30 \mathrm{GeV}$ with thermal annihilation cross-section is also consistent with the results obtained from previous studies [102]. This method can also be used to analyze the radial emission profile of a galaxy cluster. However, this requires radio data obtained from a radio telescope with a very high-resolution power (e.g., the Very-Large-Array or future SKA) because the distance of a galaxy cluster is very large.

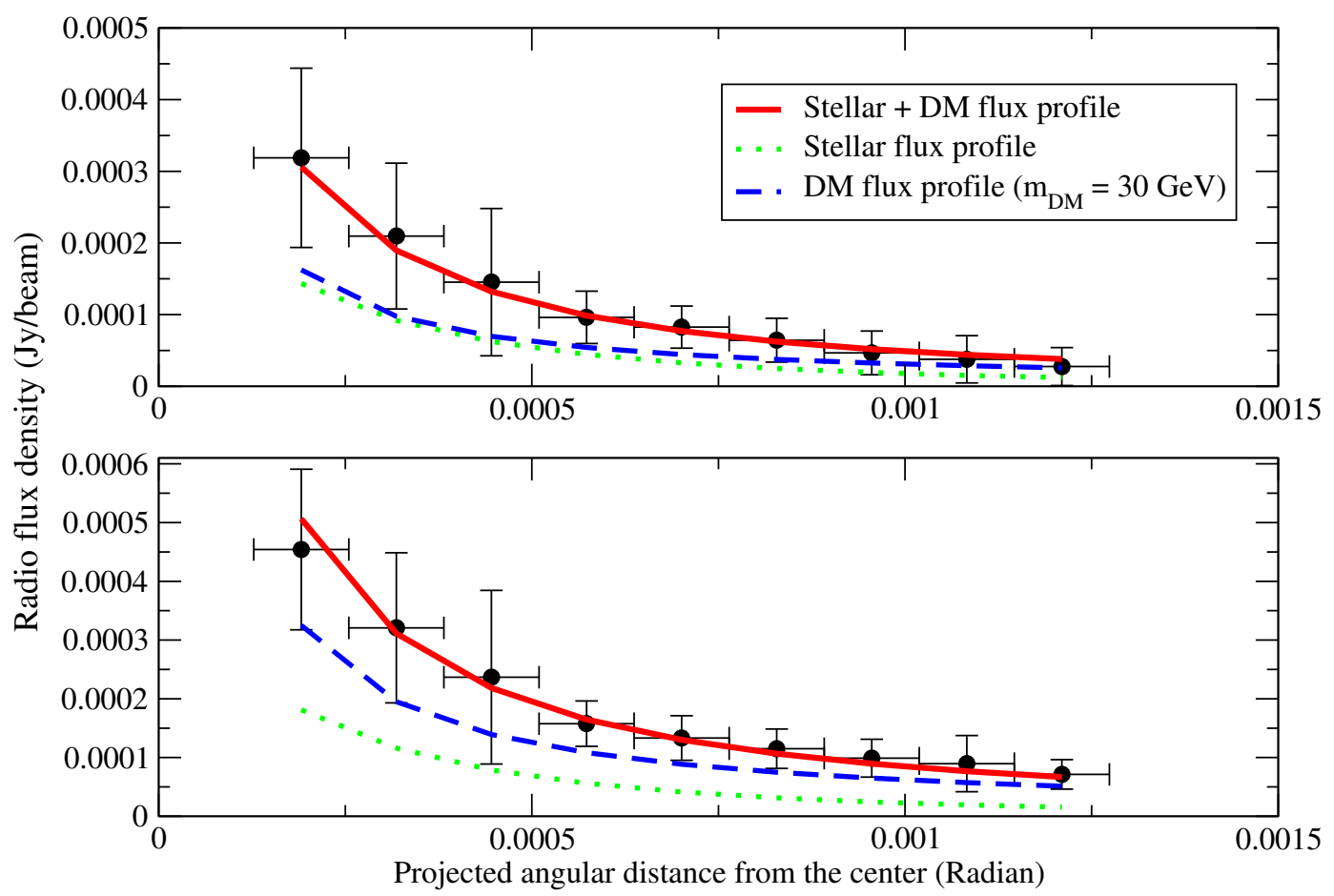

Figure 5. The best-fit thermal dark matter scenario for the radio emission profile of the M31 galaxy (assumed $m_{D M}=30 \mathrm{GeV}$ ) [102]. The red solid line, green dotted line, and blue dashed line represent the total predicted radio flux profile $S(\theta)$, the stellar flux contribution, and the dark matter contribution, respectively (upper: $8.35 \mathrm{GHz}$; lower: $4.85 \mathrm{GHz}$ ). The radio data were extracted from [62].

In fact, most of our previous discussion focused on the WIMP dark matter, which has the mass ranging from $\sim \mathrm{GeV}-\mathrm{TeV}$. It is also possible that dark matter consists of very light particles such as axions. The recent misalignment production model of axions predicts that the axion mass should be $m_{D M}=19-23 \mu \mathrm{eV}$ if axions are all cold dark matter [103]. Theoretically, axions could decay into photons spontaneously with a specific energy $E=m_{D M} c^{2} / 2$. The spontaneous decay of axions in the above-predicted mass range gives the emission frequency $v=m_{D M} c^{2} / 2 h \approx 2.3-2.8 \mathrm{GHz}$ (spontaneous emission, not synchrotron emission), which coincides with most of the observing frequencies of radio telescopes. However, the signal of axions is not easy to detect because the interaction between axions and the standard model particles is very small. The coupling constant between axion and photon interaction can be as small as $10^{-11} \mathrm{GeV}^{-1}$ [104], which gives the decay time of an axion to be more than $10^{40} \mathrm{~s}$ [103]. Fortunately, the spontaneous decay of axions could be greatly enhanced by the stimulated emission mechanism if the background contains a large amount of photons with the same emission frequency $[103,105]$. Therefore, detecting the decay signal of axion dark matter is still possible if we have a radio telescope with very high sensitivity. Future radio observations will be one of the crucial ways to verify the axion dark matter model. 


\section{Conclusions}

Radio observations have a very long tradition in astrophysical and cosmological research. It is still a developing field, and many state-of-the-art radio telescopes are providing service or being built. It has a great potential for radio observations to contribute to some great advances in the knowledge of our universe and breakthroughs in our understanding of physics. In this article, we focused on and reviewed a particular area, which uses radio observational data to constrain dark matter properties. Self-annihilation of WIMP dark matter or spontaneous decay of axion dark matter can produce radio emissions so that we can perform analyses based on the radio signals received. The theoretical framework of the dark matter annihilation model is well developed. Using radio data will be one of the major ways to constrain dark matter properties or search for dark matter signals, which is complementary to the gamma-ray, cosmic-ray, and neutrino analyses. We anticipate that this field of research will be a long-lasting and intriguing field in dark matter astrophysics.

Funding: This research was funded by the Research Grants Council of the Hong Kong Special Administrative Region, China (Grant No. EdUHK 28300518).

Institutional Review Board Statement: Not applicable.

Informed Consent Statement: Not applicable.

Data Availability Statement: Not applicable.

Conflicts of Interest: The author declares no conflict of interest.

\section{References}

1. Bertone, G.; Bozorgnia, N.; Kim, J.S.; Liem, S.; McCabe, C.; Otten, S.; de Austri, R.R. Identifying WIMP dark matter from particle and astroparticle data. J. Cosmol. Astropart. Phys. 2018, 3, 26. [CrossRef]

2. Calore, F.; Cholis, I.; McCabe, C.; Weniger, C. A tale of tails: Dark matter interpretations of the Fermi GeV excess in light of background model systematics. Phys. Rev. D 2015, 91, 063003. [CrossRef]

3. Abdo, A.A.; Ackermann, M.; Ajello, M.; Atwood, W.B.; Axelsson, M.; Baldini, L.; Lovellette, M.N.; Fermi-LAT Collaboration. Searching for dark matter annihilation from Milky Way dwarf spheroidal galaxies with six years of Fermi Large Area Telescope data. Phys. Rev. Lett. 2015, 115, 231301.

4. Daylan, T.; Finkbeiner, D.P.; Hooper, D.; Linden, T.; Portillo, S.K.N.; Rodd, N.L.; Slatyer, T.R. The characterization of the gamma-ray signal from the central Milky Way: A case for annihilating dark matter. Phys. Dark Uni. 2016, 12, 1. [CrossRef]

5. Abazajian, K.N.; Keeley, R.E. Bright gamma-ray Galactic Center excess and dark dwarfs: Strong tension for dark matter annihilation despite Milky Way halo profile and diffuse emission uncertainties. Phys. Rev. D 2016, 93, 083514. [CrossRef]

6. Albert, A.; Fermi-LAT, DES Collaborations. Searching for dark matter annihilation in recently discovered Milky Way satellites with Fermi-Lat. Astrophys. J. 2017, 834, 110. [CrossRef]

7. Chan, M.H.; Leung, C.H. Ruling out dark matter interpretation of the galactic GeV excess by gamma-ray data of galaxy clusters. Sci. Rept. 2017, 7, 14895. [CrossRef]

8. Aguilar, M.; Alberti, G.; Alpat, B.; Alvino, A.; Ambrosi, G.; Andeen, K.; Anderhub, H.; Arruda, L.; Azzarello, P.; Bachlechner, A.; et al. First result from the Alpha Magnetic Spectrometer on the International Space Station: Precision measurement of the positron fraction in primary cosmic rays of 0.5-350 GeV. Phys. Rev. Lett. 2013, 110, 141102. [CrossRef]

9. Ambrosi, G.; An, Q.; Asfandiyarov, R.; Azzarello, P.; Bernardini, P.; Bertucci, B.; Cai, M.S.; Chang, J.; Chen, D.Y.; Chen, H. F.; et al. Direct detection of a break in the teraelectronvolt cosmic-ray spectrum of electrons and positrons. Nature 2017, $552,63$.

10. Aguilar, M.; Ali Cavasonza, L.; Ambrosi, G.; Arruda, L.; Attig, N.; Azzarello, P.; Bachlechner, A.; Barao, F.; Barrau, A.; Barrin, L.; et al. Towards understanding the origin of cosmic-ray positrons. Phys. Rev. Lett. 2019, 122, 041102. [CrossRef]

11. Chan, M.H.; Lee, C.M. Origin of the DAMPE $1.4 \mathrm{TeV}$ peak. Mon. Not. R. Astron. Soc. 2019, 486, L85. [CrossRef]

12. Ajello, M.; Baldini, L.; Ballet, J.; Barbiellini, G.; Bastieri, D.; Bellazzini, R.; Bissaldi, E.; Blandford, R.D.; Bloom, E.D.; Bottacini, E.; et al. Characterizing the population of pulsars in the inner Galaxy with the Fermi-Large Area Telescope. arXiv 2017, arXiv:1705.00009.

13. Bartels, R.; Hooper, D.; Linden, T.; Mishra-Sharma, S.; Rodd, N.L.; Safdi, B.R.; Slatyer, T.R. Comment on “Characterizing the population of pulsars in the Galactic bulge with the Fermi large area telescope". Phys. Dark Univ. 2018, 20, 88. [CrossRef]

14. Blasi, P.; Olinto, A.V.; Tyler, C. Detecting WIMPs in the microwave sky. Astropart. Phys. 2003, 18, 649. [CrossRef]

15. Aloisio, R.; Blasi, P.; Olinto, A.V. Neutralino annihilation at the galactic centre revisited. J. Cosmol. Astropart. Phys. $2004,5,7$. [CrossRef] 
16. Tasitsiomi, A.; Siegal-Gaskins, J.M.; Olinto, A.V. Gamma-ray and synchrotron emission from neutralino annihilation in the Large Magellanic Cloud. Astropart. Phys. 2004, 21, 637. [CrossRef]

17. Baltz, E.A.; Wai, L. Diffuse inverse Compton and synchrotron emission from dark matter annihilations in galactic satellites. Phys. Rev. D 2004, 70, 023512. [CrossRef]

18. Borriello, E.; Cuoco, A.; Miele, G. Radio constraints on dark matter annihilation in the galactic halo and its substructures. Phys. Rev. D 2009, 79, 023518. [CrossRef]

19. Siffert, B.B.; Limone, A.; Borriello, E.; Longo, G.; Miele, G. Radio emission from dark matter annihilation in the Large Magellanic Cloud. Mon. Not. R. Astron. Soc. 2011, 410, 2463. [CrossRef]

20. Natarajan, A.; Peterson, J.B.; Voytek, T.C.; Spekkens, K.; Mason, B.; Aguirre, J.; Willman, B. Bounds on dark matter properties from radio observations of Ursa Major II using the Green Bank Telescope. Phys. Rev. D 2013, 88, 083535. [CrossRef]

21. Cholis, I.; Hooper, D.; Linden, T. A critical reevaluation of radio constraints on annihilating dark matter. Phys. Rev. D 2015, 91, 083507. [CrossRef]

22. Regis, M.; Colafrancesco, S.; Profumo, S.; de Blok, W.J.G.; Massardi, M.; Richter, L. Local Group dSph radio survey with ATCA (III): Constraints on particle dark matter. J. Cosmol. Astropart. Phys. 2014, 10, 016. [CrossRef]

23. Natarajan, A.; Aguirre, J.E.; Spekkens, K.; Mason, B.S. Green Bank Telescope constraints on dark matter annihilation in Segue I. arXiv 2015, arXiv:1507.03589.

24. Regis, M.; Richter, L.; Colafrancesco, S. Dark matter in the Reticulum II dSph: A radio search. J. Cosmol. Astropart. Phys. 2017, 07, 025. [CrossRef]

25. Chan, M.H. Relieving tensions related to the dark matter interpretation of the Fermi-LAT data. Galaxies 2018, 6, 92. [CrossRef]

26. Vollmann, M.; Heesen, V.; Shimwell, T.W.; Hardcastle, M.J.; Brüggen, M.; Sigl, G.; Röttgering, H.J.A. Radio constraints on dark matter annihilation in Canes Venatici I with LOFAR. Mon. Not. R. Astron. Soc. 2020, 496, 2663. [CrossRef]

27. Egorov, A.E.; Pierpaoli, E. Constraints on dark matter annihilation by radio observations of M31. Phys. Rev. D 2013, 88, 023504. [CrossRef]

28. Chan, M.H. Revisiting the constraints on annihilating dark matter by the radio observational data of M31. Phys. Rev. D 2016, 94, 023507. [CrossRef]

29. Chan, M.H.; Cui, L.; Liu, J.; Leung, C.S. Ruling out $\sim 100-300 \mathrm{GeV}$ thermal relic annihilating dark matter by radio observation of the Andromeda galaxy. Astrophys. J. 2019, 872, 177. [CrossRef]

30. Borriello, E.; Longo, G.; Miele, G.; Paolillo, M.; Siffert, B.B.; Tabatabaei, F.S.; Beck, R. Searching for dark matter in Messier 33. Astrophys. J. 2010, 709, L32. [CrossRef]

31. Chan, M.H. Constraining annihilating dark matter by radio data of M33. Phys. Rev. D 2017, 96, 043009. [CrossRef]

32. Chan, M.H. A possible signature of annihilating dark matter. Mon. Not. R. Astron. Soc. 2018, 474, 2576. [CrossRef]

33. Chan, M.H. A new target object for constraining annihilating dark matter. Astrophys. J. 2017, 844, 9. [CrossRef]

34. Colafrancesco, S.; Profumo, S.; Ullio, P. Multi-frequency analysis of neutralino dark matter annihilations in the Coma cluster. Astron. Astrophys. 2006, 455, 21. [CrossRef]

35. Storm, E.; Jeltema, T.E.; Profumo, S.; Rudnick, L. Constraints on dark matter annihilation in clusters of galaxies from diffuse radio emission. Astrophys. J. 2013, 768, 106. [CrossRef]

36. Colafrancesco, S.; Marchegiani, P.; Beck, G. Evolution of darak matter halos and their radio emissions. J. Cosmol. Astrophys. Phys. 2015, 2, 032. [CrossRef]

37. Chan, M.H.; Lee, C.M. Fitting dark matter mass with the radio continuum spectral data of the Ophiuchus cluster. Phys. Dark Uni. 2019, 26, 100355. [CrossRef]

38. Chan, M.H.; Lee, C.M.; Ng, C.-Y.; Leung, C.S. Constraining annihilating dark matter mass by the radio continuum spectral data of a high-redshift galaxy cluster. Astrophys. J. 2020, 900, 126. [CrossRef]

39. Chan, M.H.; Lee, C.M. A possible radio signal of annihilating dark matter in the Abell 4038 cluster. Mon. Not. R. Astron. Soc. 2021, 500, 5583. [CrossRef]

40. Fornengo, N.; Lineros, R.; Regis, M.; Taoso, M. Possibility of a dark matter interpretation for the excess in isotropic radio emission reported by ARCADE. Phys. Rev. Lett. 2011, 107, 271302. [CrossRef]

41. Cirelli, M.; Corcella, G.; Hektor, A.; Hütsi, G.; Kadastik, M.; Panci, P.; Raidal, M.; Sala, F.; Strumia, A. PPPC 4 DM ID: A poor particle physicist cookbook for dark matter indirect detection. J. Cosmol. Astropart. Phys. 2011, 3, 051. [CrossRef]

42. Atoyan, A.M.; Aharonian, F.A.; Völk, H.J. Electrons and positrons in the galactic cosmic rays. Phys. Rev. D 1995, $52,3265$. [CrossRef] [PubMed]

43. Strong, A.W.; Moskalenko, I.V. Propagation of cosmic-ray nucleons in the Galaxy. Astrophys. J. 1998, 509, 212. [CrossRef]

44. Evoli, C.; Gaggero, D.; Grasso, D.; Maccione, L. Cosmic ray nuclei, antiprotons and gamma rays in the galaxy: A new diffusion model. J. Cosmol. Astropart. Phys. 2008, 10, 18. [CrossRef]

45. Kissmann, R. PICARD: A novel code for the Galactic Cosmic Ray propagation problem. Astropart. Phys. 2014, 55, 37. [CrossRef]

46. Maurin, D. USINE: Semi-analytical models for Galactic cosmic-ray propagation. Comp. Phys. Comm. 2020, 247, 106942. [CrossRef]

47. Kar, A.; Mitra, S.; Mukhopadhyaya, B.; Choudhury, T.R. Heavy dark matter particle annihilation in dwarf spheroidal galaxies: Radio signals at the SKA telescope. Phys. Rev. D 2020, 101, 023015. [CrossRef]

48. Profumo, S.; Ullio, P. Particle Dark Matter: Observations, Models and Searches; Bertone, G., Ed.; Cambridge University Press: Cambridge, UK, 2010; Chapter 27. 
49. Bertone, G.; Cirelli, M.; Strumia, A.; Taoso, M. Gamma-ray and radio tests of the $e^{ \pm}$excess from DM annihilations. J. Cosmol. Astropart. Phys. 2009, 3, 009. [CrossRef]

50. Ullio, P.; Valli, M. A critical reassessment of particle dark matter limits from dwarf satellites. J. Cosmol. Astropart. Phys. 2016, 7, 025. [CrossRef]

51. Steigman, G.; Dasgupta, B.; Beacom, J.F. Precise relic WIMP abundance and its impact on searches for dark matter annihilation. Phys. Rev. D 2012, 86, 023506. [CrossRef]

52. Sommerfeld, A. Öber die Beugung und Bremsung der Elektronen. Ann. Der Phys. 1931, 403, 257. [CrossRef]

53. Feldman, D.; Liu, Z.; Nath, P. PAMELA positron excess as a signal from the hidden sector. Phys. Rev. D 2009, $79,063509$. [CrossRef]

54. Kim, H.; Hong, J.-P.; Shin, C.S. A map of the non-thermal WIMP. Phys. Lett. B 2017, 768, 292. [CrossRef]

55. Visinelli, L. (Non-)thermal production of WIMPs during Kination. Symmetry 2018, 10, 546. [CrossRef]

56. Choi, K.-Y.; Kang, S.K.; Kim, J. Non-thermal WIMP baryogenesis. Phys. Lett. B 2018, 782, 657. [CrossRef]

57. Navarro, J.F.; Frenk, C.S.; White, S.D.M. A universal density profile from hierarchical clustering. Astrophys. J. 1997, $490,493$. [CrossRef]

58. Chen, Y.; Reiprich, T.H.; Böhringer, H.; Ikebe, Y.; Zhang, Y.-Y. Statistics of X-ray observables for the cooling-core and non-cooling core galaxy clusters. Astron. Astrophys. 2007, 466, 805. [CrossRef]

59. Molinè, A.; Sànchez-Conde, M.A.; Palomares-Ruiz, S.; Prada, F. Characterization of subhalo structural properties and implications for dark matter annihilation signals. Mon. Not R. Astron. Soc. 2017, 466, 4974. [CrossRef]

60. Sànchez-Conde, M.A.; Prada, F. The flattening of the concentration-mass relation towards low halo masses and its implications for the annihilation signal boost. Mon. Not. R. Astron. Soc. 2014, 442, 2271. [CrossRef]

61. Sofue, Y. Dark halos of M31 and the Milky Way. Pub. Astron. Soc. Jpn. 2015, 67, 75. [CrossRef]

62. Gießübel, R.; Beck, R. The magnetic field structure of the central region in M31. Astron. Astrophys. 2014, 571, A61. [CrossRef]

63. Govoni, F.; Murgia, M.; Vacca, V.; Loi, F.; Girardi, M.; Gastaldello, F.; Giovannini, G.; Feretti, L.; Paladino, R.; Carretti, E.; et al. Sardinia Radio Telescope observations of Abell 194. The intra-cluster magnetic field power spectrum. Astron. Astrophys. 2017, 603, A122. [CrossRef]

64. Kunz, M.W.; Schekochihin, A.A.; Cowley, S.C.; Binney, J.J.; Sanders, J.S. A thermally stable heating mechanism for the intracluster medium: Turbulence, magnetic fields and plasma instabilities. Mon. Not. R. Astron. Soc. 2011, 410, 2446. [CrossRef]

65. Hooper, D.; Finkbeiner, D.P.; Dobler, G. Possible evidence for dark matter annihilations from the excess microwave emission around the center of the Galaxy seen by the Wilkinson Microwave Anisotropy Probe. Phys. Rev. D 2007, 76, 083012. [CrossRef]

66. Carlson, E.; Hooper, D.; Linden, T.; Profumo, S. Testing the dark matter origin of the WMAP-Planck haze with radio observations of spiral galaxies. J. Cosmol. Astropart. Phys. 2013, 7, 026. [CrossRef]

67. Regis, M.; Ullio, P. Multiwavelength signals of dark matter annihilations at the Galactic center. Phys. Rev. D 2008, 78, 043505. [CrossRef]

68. Wechakama, M.; Ascasibar, Y. Multimessenger constraints on dark matter annihilation into electron-positron pairs. Mon. Not. R. Astron. Soc. 2014, 439, 566. [CrossRef]

69. Gondolo, P.; Silk, J. Dark matter annihilation at the Galactic Center. Phys. Rev. Lett. 1999, 83, 1719. [CrossRef]

70. Fields, B.D.; Shapiro, S.L.; Shelton, J. Galactic Center gamma-ray excess from dark matter annihilation: Is there a black hole spike? Phys. Rev. Lett. 2014, 113, 151302. [CrossRef]

71. Egorov, A.E.; Gaskins, J.M.; Pierpaolo, E.; Pietrobon, D. Dark matter implications of the WMAP-Planck Haze. J. Cosmol. Astropart. Phys. 2016, 3, 060. [CrossRef]

72. Gießübel, R.; Heald, G.; Beck, R.; Arshakian, T. Polarized synchrotron radiation from the Andromeda galaxy M31 and background sources at $350 \mathrm{MHz}$. Astron. Astrophys. 2013, 559, A27. [CrossRef]

73. Chan, M.H.; Lee, C.M. Constraining the annihilating dark matter mass by the radio continuum spectral data of the NGC4214 galaxy. Phys. Rev. D 2020, 102, 063017. [CrossRef]

74. Fang, K.; Linden, T. Cluster mergers and the origin of the ARCADE-2 excess. J. Cosmol. Astropart. Phys. 2016, 10, 004. [CrossRef]

75. Kehayias, J.; Kephart, T.W.; Weiler, T.J. The excess radio background and fast radio transients. J. Cosmol. Astropart. Phys. 2015, 10, 053. [CrossRef]

76. Fairbairn, M.; Grothaus, P. Note on the dark matter explanation of the ARCADE excess and AMS data. Phys. Rev. D 2014, 90, 127302. [CrossRef]

77. Evoli, C.; Morlino, G.; Blasi, P.; Aloisio, R. AMS-02 beryllium data and its implication for cosmic ray transport. Phys. Rev. D 2020, 101, 023013. [CrossRef]

78. Fang, K.; Bi, X.-J.; Yin, P.-F. DAMPE proton spectrum indicates a slow-diffusion zone in the nearby ISM. Astrophys. J. 2020, 903, 69. [CrossRef]

79. Beck, G. An excess of excesses examined via dark matter radio emissions from galaxies. J. Cosmol. Astropart. Phys. 2019, 8, 019. [CrossRef]

80. Gao, L.; Frenk, C.S.; Jenkins, A.; Springel, V.; White, S.D.M. Where will supersymmetric dark matter first be seen? Mon. Not. R. Astron. Soc. 2012, 419, 1721. [CrossRef]

81. Dolag, K.; Schindler, S.; Govoni, F.; Feretti, L. Correlation of the magnetic field and the intra-cluster gas density in galaxy clusters. Astron. Astrophys. 2001, 378, 777. [CrossRef] 
82. Jaffe, W.J. Origin and transport of electrons in the halo radio source in the Coma cluster. Astrophys. J. 1977, 212, 1. [CrossRef]

83. Rephaeli, Y. Spatial distribution of Compton-produced X-ray flux from rich and regular clusters of galaxies. Astrophys. J. 1977, 212, 608. [CrossRef]

84. Rephaeli, Y. Relativistic electrons in the intracluster space of clusters of galaxies: The hard X-ray spectra and heating of the gas. Astrophys. J. 1979, 227, 364. [CrossRef]

85. Dennison, B. Formation of radio halos in clusters of galaxies from cosmic-ray protons. Astrophys. J. 1980, 239, L93. [CrossRef]

86. Keshet, U.; Loeb, A. Using radio halos and minihalos to measure the distributions of magnetic fields and cosmic rays in galaxy clusters. Astrophys. J. 2010, 722, 737. [CrossRef]

87. Bell, A.R. The acceleration of cosmic rays in shock fronts - I. Mon. Not. R. Astron. Soc. 1978, 182, 147. [CrossRef]

88. Malkov, M.A.; Drury, L.O. Nonlinear theory of diffusive acceleration of particles by shock waves. Rep. Prog. Phys. 2001, 64, 429. [CrossRef]

89. Schlickeiser, R.; Sievers, A.; Thiemann, H. The diffuse radio emission from the Coma cluster. Astron. Astrophys. 1987, $182,21$.

90. Thierbach, M.; Klein, U.; Wielebinski, R.R. The diffuse radio emission from the Coma cluster at $2.675 \mathrm{GHz}$ and $4.85 \mathrm{GHz}$. Astron. Astrophys. 2003, 397, 53. [CrossRef]

91. Brunetti, G.; Setti, G.; Feretti, L.; Giovannini, G. Particle reacceleration in the Coma cluster: Radio properties and hard X-ray emission. Mon. Not. R. Astron. Soc. 2001, 320, 365. [CrossRef]

92. Enßlin, T.A.E.; Gopal-Krishna. Reviving fossil radio plasma in clusters of galaxies by adiabatic compression in environmental shock waves. Astron. Astrophys. 2001, 366, 26. [CrossRef]

93. Enßlin, T.A.E.; Brüggen, M. On the formation of cluster radio relics. Mon. Not. R. Astron. Soc. 2002, 331, 1011. [CrossRef]

94. van Weeren, R.J.; de Gasperin, F.; Akamatsu, H.; Brüggen, M.; Feretti, L.; Kang, H.; Stroe, A.; Zandanel, F. Diffuse radio emission from galaxy clusters. Sp. Sci. Rev. 2019, 215, 16. [CrossRef]

95. Murgia, M.; Eckert, D.; Govoni, F.; Ferrari, C.; Pandey-Pommier, M.; Nevalainen, J.; Paltani, S. GMRT observations of the Ophiuchus galaxy cluster. Astron. Astrophys. 2010, 514, A76. [CrossRef]

96. Macario, G.; Venturi, T.; Intema, H.T.; Dallacasa, D.; Brunetti, G.; Cassano, R.; Giacintucci, S.; Ferrari, C.; Ishwara-Chandra, C.H.; Athreya, R. 153 MHz GMRT follow-up of steep-spectrum diffuse emission in galaxy clusters. Astron. Astrophys. 2013, 551, A141. [CrossRef]

97. Kale, R.; Dwarakanath, K.S. Multi-frequency studies of radio relics in the galaxy clusters A4038, A1664, and A786. Astrophys. J. 2012, 744, 46. [CrossRef]

98. Brown, A.M.; Lacroix, T.; Lloyd, S.; Hm, C.B.; Chadwick, P. Understanding the $\gamma$-ray emission from the globular cluster 47 Tuc: Evidence for dark matter? Phys. Rev. D 2018, 98, 041301. [CrossRef]

99. Cholis, I.; Linden, T.; Hooper, D. A robust excess in the cosmic-ray antiproton spectrum: Implications for annihilating dark matter. Phys. Rev. D 2019, 99, 103026. [CrossRef]

100. Storm, E.; Jeltema, T.E.; Splettstoesser, M.; Profumo, S. Synchrotron emission from dark matter annihilation: Predictions for constraints from non-detections of galaxy clusters with new radio surveys. Astrophys. J. 2017, 839, 33. [CrossRef]

101. Bartels, R.; Storm, E.; Weniger, C.; Calore, F. The Fermi-LAT GeV excess as a tracer of stellar mass in the Galactic bulge. Nat. Astron. 2018, 2, 819. [CrossRef]

102. Chan, M.H.; Yeung, C.F.; Cui, L.; Leung, C.S. Analysing the radio flux density profile of the M31 galaxy: A possible dark matter interpretation. Mon. Not. R. Astron. Soc. 2021, 501, 5692-5696

103. Battye, R.A.; Garbrecht, B.; McDonald, J.I.; Pace, F.; Srinivasan, S. Dark matter axion detection in the radio/mm waveband. Phys. Rev. D 2020, 102, 023504. [CrossRef]

104. Graham, P.W.; Irastorza, I.G.; Lamoreaux, S.K.; Lindner, A.; van Bibber, K.A. Experimental searches for the axion and axion-like particles. Ann. Rev. Nucl. Part. Sci. 2015, 65, 485. [CrossRef]

105. Caputo, A.; Garay, C.P.; White, S.J. Looking for axion dark matter in dwarf spheroidal galaxies. Phys. Rev. D 2018, $98,083024$. [CrossRef] 